\title{
The Cytomorphological Characteristics of Non-small Cell Lung Cancer are Associated with Its Radiological Features
}

Ryota Tanaka $^{1 *}$, Norihiko Sakamoto², Hitomi Suzuki ${ }^{2}$, Keisei Tachibana ${ }^{1}$, Hidefumi Takei ${ }^{1}$, Koji Kishimoto ${ }^{2}$ Masachika Fujiwara ${ }^{2}$, Hiroshi Kamma², Junji Shibahara² and Haruhiko Kondo'

${ }^{1}$ Department of Surgery, School of Medicine, Kyorin University, Tokyo, Japan

${ }^{2}$ Department of Pathology, School of Medicine, Kyorin University, Tokyo, Japan

\begin{abstract}
Background: Distinguishing the histological subtype of lung cancer is extremely important because chemotherapy agents may have different chemotherapeutic effects in different histological subtypes. This study retrospectively evaluated the characteristic differences in the cytomorphological and radiological features of nonsmall cell lung cancer.

Methods: This study population included 50 lung cancer patients who were diagnosed based on bronchoscopic biopsy findings and underwent surgery from April 2011 to May 2016. Cyotological specimens (Papanicolaou's staining) were separately analyzed by two experienced cytotechnicians. The associations between the clinicopathological data, as well as the preoperative radiological features on computed tomography (CT) and positron emission tomography (PET) and cytomorphological analyses were analyzed.

Results: Forty-five specimens were analyzed (adenocarcinoma [ADC] [ $n=38]$, squamous cell carcinomas [SQCC] [ $n=7])$. The ADCs were cytomorphologically sub-classified as follows: acinar $(n=25)$, solid $(n=10)$, lepidic $(n=2)$ and papillary $(n=1)$. Among specimens with a solid pattern of $A D C$, there was a significantly greater percentage of samples with a necrotic background $(50 \%)$, predominant 3D clusters $(100 \%)$ and conspicuous nucleoli $(80 \%)$ compared to specimens with a nonsolid pattern of ADC. The CT images of patients with solid ADC (90\%) and SQCC $(100 \%)$ tended to show solid nodules on CT. On PET, the maximum standardized uptake values at 120 minutes in solid ADC and SQCC were significantly higher in comparison to nonsolid ADC $(12.8 \pm 6.1$ and $17.2 \pm 7.3$ versus $8.9 \pm 4.4$, respectively)
\end{abstract}

Conclusions: The differentiation of the cytomorphological characteristics might predict the values of radiological features, and thereby provide information that can be used when determining the treatment strategies.

Keywords: High resolution computed tomography; Positron emission tomography; Non-small cell lung cancer; Cytomorphological subtypes; Lung adenocarcinoma

\section{Introduction}

Lung cancer is the most frequent type of major cancer worldwide. Lung cancer is histopathologically divided into small cell carcinoma and non-small cell lung cancer (NSCLC). In, addition, the discrimination of the histological subtype in NSCLC is regarded as highly important when choosing chemotherapy agents, as their effects differ according to the histological subtype [1]. The 2015 World Health Organization (WHO) classification1 reflects the 2011 International Association for the Study of Lung Cancer (IASLC)/American Thoracic Society (ATS)/ European Respiratory Society (ERS) classifications [2]. The guidelines recommend the classification of the major adenocarcinoma (ADC) subtypes according to the predominant histological pattern (primary pattern), and the estimation of the percentage of the other ADC patterns that may be present in a resected specimen (in increments of 5\%; secondary pattern). Some investigators have used analyzed the impact of the sub-classification on the prognosis of the major subtypes of resected lung ADC, which include acinar, solid, papillary, lepidic and micropapillary [3-5]. In those studies, solid and micropapillary predominant ADC were shown to have significantly worse prognoses than the other predominant subtypes; thus, they were considered to have significant value in predicting death and recurrence in the IASLC/ATS/ERS ADC classification system. Rodriguaez et al. [6,7] investigated the subtyping of ADC according to the proposed IASLC/ ATS/ERS classification based on cytology samples and published articles. On the other hand, there have been many efforts to stratify the lesions of lung ADC radiologically, using imaging modalities such as high resolution computed tomography (HRCT), magnetic resonance imaging and $[18 \mathrm{~F}]$ fluorodeoxyglucose (FDG)-positron emission tomography with an integrated computed tomography scanner (PET/CT), because the features could provide information about pathological invasiveness of the tumor, which could assist in decision-making with regard to treatment. Moreover, studies on PET have shown that the maximum standardized uptake value (SUV max) is an indicator of the potential for cancer cell proliferation, and the increased expression of cell proliferative biomarkers is associated with a worse prognosis in lung cancer $[8,9]$. Our previous study revealed that diffusion weighted imaging, which is one of the MRI sequences, might reflect the histological and cytomorphological characteristics of tumor cells $[10,11]$, while PET only reflected poorly differentiated cancer cells in surgically resected ADC [12]. The purpose of this study was to retrospectively evaluate the characteristic differences between

*Corresponding author: Tanaka R, Department of Surgery, Kyorin University School of Medicine, 6-20-2, Shinkawa, Mitaka-shi, Tokyo 181-8611, Japan, Tel: +81-422-47-5511; Fax: +81-422-44-3576; E-mail: ryota@ks.kyorin-u.ac.jp

Received October 10, 2017; Accepted November 04, 2017; Published November 12,2017

Citation: Tanaka R, Sakamoto N, Suzuki H, Tachibana K, Takei H, et al. (2017) The Cytomorphological Characteristics of Non-small Cell Lung Cancer are Associated with Its Radiological Features. J Cytol Histol 8: 482. doi: 10.4172/21577099.1000482

Copyright: @ 2017 Tanaka R, et al. This is an open-access article distributed under the terms of the Creative Commons Attribution License, which permits unrestricted use, distribution, and reproduction in any medium, provided the original author and source are credited. 
Citation: Tanaka R, Sakamoto N, Suzuki H, Tachibana K, Takei H, et al. (2017) The Cytomorphological Characteristics of Non-small Cell Lung Cancer are Associated with Its Radiological Features. J Cytol Histol 8: 482. doi: 10.4172/2157-7099.1000482

the cytomorphological findings with the histological subtypes of the bronchoscopic materials and the radiological features of HRCT and PET in the preoperative setting based on our database.

\section{Materials and Methods}

Fifty lung cancer patients with peripheral lung nodules who were diagnosed based on the examination of bronchoscopic biopy specimens underwent surgery in our department of Kyorin University Hospital from April 2011 to December 2016. The study received institutional review board approval, and all patients provided their written informed consent to participate in the study. The patients were classified based on the clinical and pathological stage, according to the International System for Staging Lung Cancer [13]. Endobronchial ultrasonography with a guide-sheath (EBUS-GS) was routinely performed to obtain small tissue specimens and cytological materials from peripheral lung nodules with a solid component. The cyotological material obtained by bronchoscopic brushing was placed onto a glass slide, immediately fixed in $95 \%$ ethanol, and subjected to Papanicolaou staining. Cytomorphological studies (including histological typing, subtyping of ADC and the architectural and nuclear features) were performed retrospectively using the criteria reported by Rodriguaez et al. [7] The specimens were separately observed on different microscopes by two experienced cytotechnicians, (N.S. and H.S., with 24 and 10 years of experience in cytopathology, respectively). The cytotechnicians were blinded to the histopathological diagnosis. The final judgement of the studies was confirmed if the two opinions corresponded; if the two opinions did not correspond, the cytotechnicians discussed the results until they were in agreement. The clinicopathological data and the preoperative radiological features of the patients were stored in our lung cancer database; information from this database was used for the comparison of the cytomorphological analyses in the present study. All patients underwent chest HRCT (2-mm slice) within 1 month before surgery as a preoperative evaluation at the department of diagnostic radiology in our hospital. HRCT images were generated using a non-enhanced multidetector CT system (Aquilion One Vision Edition version 6.0; Toshiba Medical Systems, Tokyo, Japan). The CT scan parameters were as follows: tube current, automatic exposure control SD 12; tube voltage, $120 \mathrm{kV}$; rotation speed, $0.5 \mathrm{~s} / \mathrm{rot}$; table speed, BP 0.813; reconstruction filter, FC52 AIDR Standard; window width, 600; and window level, -600, for lung window images. We used automatic exposure control, the volume helical scan mode, and multi-planar reconstruction. On HRCT images, the tumor lesions were classified into three radiological subtypes; ground-glass opacity (GGO, so-called pure GGO), part solid (GGO with consolidation) and solid (without GGO). GGO was defined as a hazy area of lung opacity with increased attenuation that was not associated with the obscuration of the underlying vessels. For the tumor size, the longest tumor diameters were measured on lung window images, and the $\mathrm{C} / \mathrm{T}$ ratio was calculated based on the diameter of consolidation divided by the diameter the tumor size on the same image. For staging, FDG-PET/ CT was performed preoperatively with an integrated PET/CT scanner (Biograph 16; Siemens Medical Solutions, Knoxville, TN) at the Tokorozawa PET Radiology Center in Saitama. All of the patients fasted for 6 hours before scanning. An emission scan was performed 60 and 120 minutes after the intravenous administration of 18F-FDG (185 to $240 \mathrm{MBq}$ ). Patients with blood glucose concentrations of $>200 \mathrm{mg} / \mathrm{dl}$ at the time of the examination were excluded from the study. The scanning time for positron emission was $3.5 \mathrm{~min}$ per image slice, and slices were obtained from the top of the skull to the mid-thigh. The images were reconstructed, and the ROI was drawn on the fusion image with the corresponding CT scan by a radiologist without any knowledge of the patient's information. In addition, the SUV max of each primary lung cancer lesion was calculated automatically at 60 and 120 minutes, after the delineation of the ROI on attenuation-corrected 18F-FDG PET/ $\mathrm{CT}$ images. The data and ratios are presented as the mean \pm standard deviation (SD). The associations of categorical variables were evaluated using the chi-squared test. The data and the age of the patients was compared in several cytological types and categories of tumors using Student's t-test. All of the statistical analyses were performed using the SPSS software program (version 19.0, IBM Corp, Chicago, IL, USA). P value of $<0.05$ were considered to indicate statistical significance.

\section{Results}

Five of the 50 cases of lung cancer were excluded (typical carcinoid, $\mathrm{n}=1$; not otherwise specified [NOS], $\mathrm{n}=4$ ). Thus, 45 specimens in 45 patients (ADC, $n=38$; SQCC, $n=7$ ) were analyzed in the present study. Thirty-eight ADCs were cytomorphologically sub-classified into the acinar $(n=25)$ (Figure 1), solid $(n=10)$ (Figure 2), lepidic $(n=2)$ and papillary $(n=1)$ subtypes. With the exception of the solid sub-type, the other subtypes were categorized as nonsolid for the statistical analyses, in accordance with the method of Rodriguaez et al. [6,7]. The cytological features, including the architectural and nuclear characteristics are summarized (according to the pattern) in Table 1. The percentages of solid pattern specimens with a necrotic background, (5 of 10, 50\%), predominant $3 \mathrm{D}$ clusters (10 of $10,100 \%)$ and conspicuous nucleoli (8 of $10,80 \%$ ) were significantly greater in comparison to the nonsolid specimens; the differences in these features were statistically significant ( $\mathrm{P}=0.007, \mathrm{P}=0.0001$ and $\mathrm{P}=0.0001$, respectively). The clinicopathological characteristics and radiological features in the patients are summarized in Table 2. There were statistically significant differences among the groups with regard to the age and smoking index. With regard to the $\mathrm{CT}$ features, the $\mathrm{C} / \mathrm{T}$ ratio of the nonsolid pattern group $(0.9 \pm 0.1)$ was significantly lower than of the subtypes (solid pattern and SQCC) (1.0 \pm 0.0 and $1.0 \pm 0.0, \mathrm{p}=0.045$ and 0.002 , respectively). The SUV max of the SQCC group at 60 and 120 minutes $(12.3 \pm 4.0$ and $17.2 \pm 7.3)$ was significantly higher than in the nonsolid pattern group $(6.8 \pm 3.4$ and $8.9 \pm 4.4 ; \mathrm{p}=0.001$ and 0.001 , respectively). The SUV max at 60 and 120 minutes in the solid pattern group was lower in comparison to the SQCC group; however, the difference was not statistically significant. In addition, the SUV max at 60 and 120 minutes in the solid pattern group

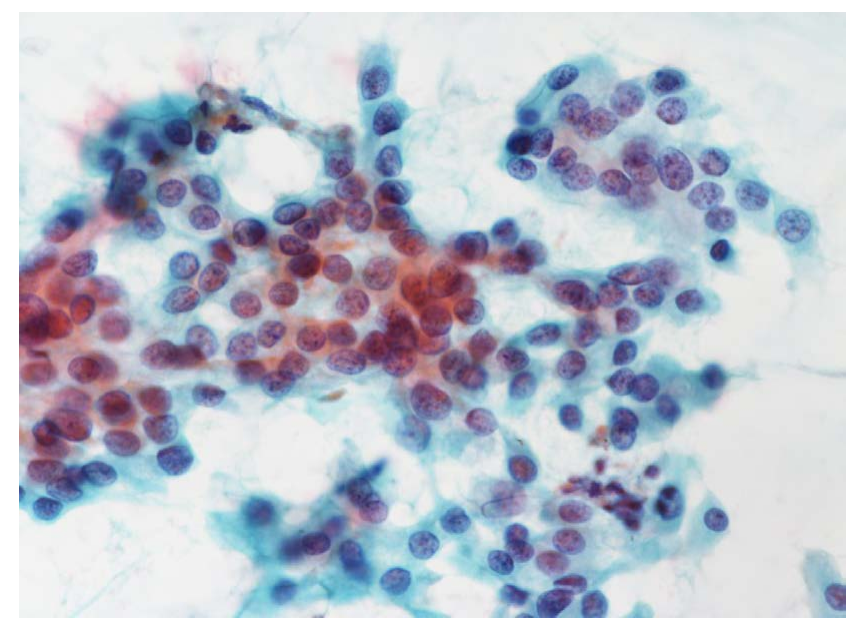

Figure 1: Lung adenocarcinoma with an acinar pattern. Clusters of tumor cells show central lumen formation and 2-dimensional sheets. Tumor cells have small nucleoli and intranuclear inclusions. (Papanicolaou staining, $\times 600$ ). 


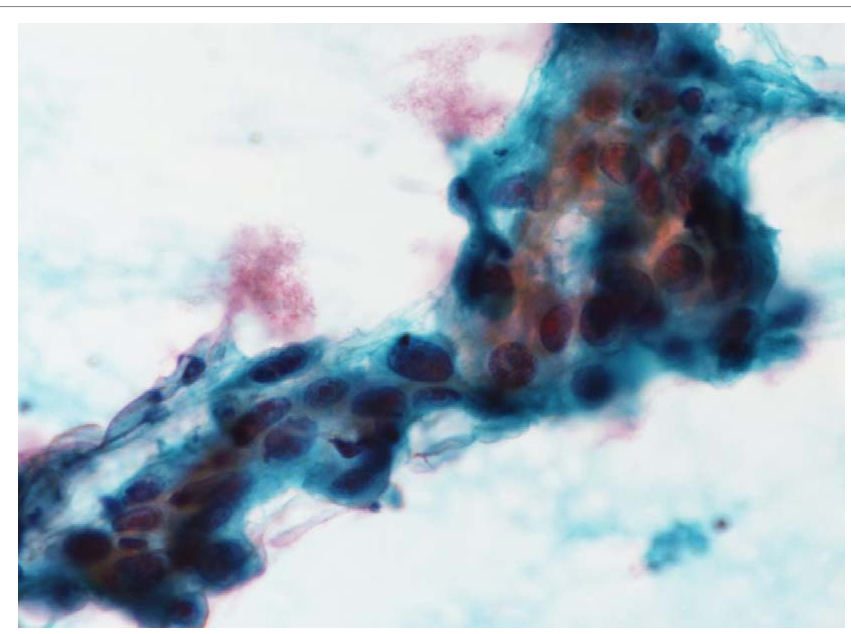

Figure 2: Lung adenocarcinoma with a solid pattern. Tumor cells with conspicuous nucleoli show 3-dimensional sheets without architectural formations (i.e., cribriform or papillary formations). (Papanicolaou staining, $\times 600$ ).

\begin{tabular}{|c|c|c|c|}
\hline Cytological features & Nonsolid $(n=28)$ & Solid $(n=10)$ & $p$ value \\
\hline \multicolumn{4}{|l|}{ Background } \\
\hline Necrosis & 2 & 5 & $0.007^{a}$ \\
\hline Clean & 12 & 1 & \\
\hline Inflammatory & 14 & 4 & \\
\hline \multicolumn{4}{|c|}{ Predominant architecture of groups } \\
\hline Flat sheets & 20 & 0 & $0.0001^{\mathrm{b}}$ \\
\hline 3D clusters & 8 & 10 & \\
\hline Papillary structures & 0 & 0 & \\
\hline \multicolumn{4}{|c|}{ Architecture: lumens or acinar pattern } \\
\hline Present & 6 & 5 & 0.087 \\
\hline Absent & 22 & 5 & \\
\hline \multicolumn{4}{|l|}{ Nuclear size } \\
\hline Small/medium & 27 & 8 & 0.098 \\
\hline Large & 1 & 1 & \\
\hline \multicolumn{4}{|l|}{ Pleomorphism } \\
\hline Present & 0 & 1 & 0.09 \\
\hline Absent & 28 & 9 & \\
\hline \multicolumn{4}{|l|}{ Nuclear contours } \\
\hline Smooth & 14 & 4 & 0.587 \\
\hline Irregular & 14 & 6 & \\
\hline \multicolumn{4}{|l|}{ Chromatin pattern } \\
\hline Fine/granular & 27 & 10 & 0.545 \\
\hline Coarse & 1 & 0 & \\
\hline \multicolumn{4}{|l|}{ Nucleoli } \\
\hline Conspicuous & 5 & 8 & $0.0001^{\mathrm{c}}$ \\
\hline Inconspicuous & 23 & 2 & \\
\hline \multicolumn{4}{|c|}{ Intranuclear inclusions } \\
\hline Present & 10 & 4 & 0.681 \\
\hline Absent & 18 & 6 & \\
\hline \multicolumn{4}{|l|}{ Nuclear grooves } \\
\hline Present & 17 & 6 & 0.968 \\
\hline Absent & 11 & 4 & \\
\hline \multicolumn{4}{|c|}{ Presence of signet ring cells } \\
\hline Present & 0 & 0 & - \\
\hline Absent & 28 & 10 & \\
\hline \multicolumn{4}{|l|}{ Presence of giant cells } \\
\hline Present & 1 & 2 & 0.098 \\
\hline Absent & 27 & 8 & \\
\hline
\end{tabular}

ADC: Adenocarcinoma; 3D: 3-dimensional; ${ }^{\text {a-c }} \mathrm{P}$ values of $<0.05$ was considered to indicate statistical significance

Table 1: Comparison of the cytomorphological features of nonsolid patterns of lung $A D C$ versus solid patterns of lung $A D C$.
$(9.7 \pm 4.6$ and $12.8 \pm 6.1)$ was higher than in the nonsolid pattern group; the SUV max at 120 min was significantly different. The relationships between various cytological findings and the radiological features with pathological stages in ADC patients are shown in Table 3. Although the cytological findings did not differ according to the pathologic stages, cases in which $3 \mathrm{D}$ clusters and conspicuous nucleoli were detected had a significantly higher C/T ratio and SUV max at 60 and 120 minutes in comparison to cases in which these findings were not detected.

\section{Discussion}

Although distinguishing the subtype of lung cancer is important when determining the treatment strategy, cytology-based subtyping is still uncommon. However the quantities of pathological materials obtained by biopsy are not always sufficient for subtyping or genotyping; thus in addition to histological specimens, cytological specimens should be used for subtyping, as this might expand treatment choices $[14,15]$. Nizzoli et al. [16] investigated the accuracy of fine needle aspiration cytology (FNAC) in NSCLC typing in comparison to histology. In their study, the cytological and histological typing was concordant in 137 of $156(88 \%)$ cases $(\mathrm{K}=0.755 ; \mathrm{p}<0.001)$, and the positive predictive value of FNAC in predicting the type of NSCLC was $92 \%$ for adenocarcinoma and $82 \%$ for squamous cell carcinoma. Thus, the cytological and histological types (based on morphological classification with conventional routine staining) closely correlated. On the other hand, after publication of the IASLC/ATS/ERS classification, the subclassification of ADC was reported to have significant prognostic value [3-5]. Rodriguaez et al. [7] attempted to distinguish the ADC subtypes using cytological specimens; they noted that architectural and nuclear features may be helpful, particularly in distinguishing the solid pattern from other patterns; however, the cytological features of the lung ADC subtypes proposed by the IASLC/ATS/ERS classification overlap. In the present study, we attempted to distinguish the ADC subtypes based on cytological characteristics (necrotic backgrounds, predominant 3D clusters and conspicuous nucleoli) and noted statistically significant differences in the features of solid pattern tumors and nonsolid tumors.

With regard to the $\mathrm{CT}$ features, the $\mathrm{C} / \mathrm{T}$ ratio of the nonsolid pattern group was significantly lower than that of the solid pattern and SQCC groups; one reason for this is that many of the nonsolid pattern lesions showed mixed patterns with GGO and/or cavitation. In other words, specimens with cytological features of solid pattern ADC and SQCC would show a solid pattern and consolidation on pretreatment radiological imaging. Chiu et al. [17] reported that solid adenocarcinomas had a significantly higher SUV max than other subtypes (papillary, acinar and lepidic), and that tumors with a solid growth pattern showed a significantly higher SUV max than those without a solid growth pattern. Thus, their results are similar to the results of the present study in which the SUV max values of solid ADC and SQCC were higher than the values in nonsolid ADC. In our previous study, [12] the SUV max of poorly differentiated ADC (the same as solid adenocarcinoma with mucin) with malignant potential was significantly higher in comparison to the other cytological subtypes (classified by Shimosato's classification [18]). Sauter et al. [19] investigated the correlation between the glucose metabolism registered by $18 \mathrm{~F}-\mathrm{FDG}$ PET/CT and tumor perfusion quantified based on volume perfusion CT and immunohistochemical markers (Ki67 and microvessel density [MVD]), which were evaluated by CD34 staining in 24 consecutive NSCLC patients. They found that the correlation between the blood flow (BF)-metabolic relationship and the total tumor volume in SQCC $(\mathrm{r}=0.762, \mathrm{p}=0.017)$ was stronger than that in ADC $(\mathrm{r}=-0.0791, \mathrm{p}=0.788)$. Thus, they concluded that the 18F-FDG uptake 


\begin{tabular}{|c|c|c|c|c|c|c|}
\hline \multirow[t]{2}{*}{ Cytological features } & \multicolumn{2}{|c|}{ ADC } & \multirow[t]{2}{*}{ SQCC $(n=7)$} & \multicolumn{3}{|c|}{$p$ value } \\
\hline & Nonsolid $(n=28)$ & Solid $(n=10)$ & & Non- vs. Solid & Non- vs. SQ & Solid vs. SQ \\
\hline \multicolumn{7}{|l|}{ Age } \\
\hline Mean (years, mean \pm SD) & $69.8 \pm 1.9$ & $63.4 \pm 2.5$ & $72.9 \pm 1.4$ & 0.071 & 0.071 & $0.01^{\mathrm{a}}$ \\
\hline \multicolumn{7}{|l|}{ Gender } \\
\hline Male & 15 & 7 & 5 & 0.366 & 0.393 & 0.949 \\
\hline Female & 13 & 3 & 2 & & & \\
\hline \multicolumn{7}{|l|}{ Smoking index } \\
\hline$\geqq 600$ & 11 & 4 & 6 & 0.968 & $0.028^{b}$ & 0.06 \\
\hline$<600$ & 17 & 6 & 1 & & & \\
\hline \multicolumn{7}{|l|}{ CT characteristics } \\
\hline GGO/Part Solid & $0 / 10$ & $0 / 1$ & $0 / 0$ & 0.124 & 0.061 & 0.389 \\
\hline Solid & 18 & 9 & 7 & & & \\
\hline \multicolumn{7}{|l|}{ CT } \\
\hline Tumor size $(\mathrm{cm}$, mean $\pm \mathrm{SD})$ & $3.2 \pm 0.7$ & $4.1 \pm 2.1$ & $3.5 \pm 1.4$ & 0.226 & 0.518 & 0.503 \\
\hline Consolidation size $(\mathrm{cm}$, mean \pm SD) & $3.0 \pm 0.7$ & $4.1 \pm 2.1$ & $3.5 \pm 1.4$ & 0.141 & 0.161 & 0.55 \\
\hline $\mathrm{C} / \mathrm{T}$ ratio $($ mean $\pm \mathrm{SD})$ & $0.9 \pm 0.1$ & $1.0 \pm 0.0$ & $1.0 \pm 0.0$ & $0.045^{c}$ & $0.002^{\mathrm{d}}$ & 0.42 \\
\hline \multicolumn{7}{|l|}{ PET } \\
\hline SUV max at 60 min $($ mean $\pm S D)$ & $6.8 \pm 3.4$ & $9.7 \pm 4.6$ & $12.3 \pm 4.0$ & 0.061 & $0.001^{\mathrm{e}}$ & 0.246 \\
\hline SUV max at 120 min $($ mean $\pm S D)$ & $8.9 \pm 4.4$ & $12.8 \pm 6.1$ & $17.2 \pm 7.3$ & $0.050^{f}$ & $0.001^{9}$ & 0.228 \\
\hline \multicolumn{7}{|l|}{ Pathologic stage } \\
\hline I/II & $13 / 3$ & $3 / 1$ & $7 / 0$ & 0.351 & $0.033^{\mathrm{h}}$ & $0.011^{i}$ \\
\hline III/IV & $11 / 1$ & $6 / 0$ & $0 / 0$ & & & \\
\hline
\end{tabular}

ADC: Adenocarcinoma; SQCC: Squamous cell carcinoma; Non-: Nonsolid; SQ: Squamous Cell Carcinoma; SD: Standard Deviation; CT: Computed Tomography; GGO: Ground Glass Opacity; C/T ratio: Consolidation/Tumor ratio (a diameter of consolidation divided by a diameter tumor size); PET: Positron Emission Tomography; SUV: Standardized Uptake Value; Pathologic stage: I (IA and IB), II (IIA and IIB), III (IIIA and IIIB); ${ }^{\text {-iI }}$ values of $<0.05$ were considered to indicate statistical significance. Table 2: The clinicopathological characteristics and radiological features of nonsolid and solid patterns of ADC and SQCC.

\begin{tabular}{|c|c|c|c|c|c|c|c|c|c|c|c|c|}
\hline \multirow[t]{2}{*}{ Cytological features } & \multicolumn{3}{|c|}{ Pathologic stage } & \multicolumn{3}{|c|}{ CT characteristics } & \multicolumn{2}{|c|}{$\mathrm{C} / \mathrm{T}$ ratio } & \multicolumn{4}{|c|}{ PET (SUV max, mean \pm SD) } \\
\hline & $\mathrm{I} / \mathrm{II}$ & III/IV & $p$ value & Part solid & Solid & $p$ value & (mean \pm SD) & $p$ value & $60 \mathrm{~min}$ & $p$ value & $120 \mathrm{~min}$ & p value \\
\hline \multicolumn{13}{|l|}{ Necrosis } \\
\hline Present $(n=7)$ & 3 & 4 & 0.566 & 1 & 6 & 0.344 & $0.97 \pm 0.07$ & 0.201 & $11.0 \pm 5.8$ & 0.144 & $14.4 \pm 6.9$ & 0.117 \\
\hline Absent $(n=31)$ & 17 & 14 & & 10 & 21 & & $0.93 \pm 0.12$ & & $6.9 \pm 3.0$ & & $9.0 \pm 4.2$ & \\
\hline \multicolumn{13}{|l|}{ 3D cluster } \\
\hline Present $(n=18)$ & 7 & 11 & 0.107 & 2 & 16 & $0.021^{\mathrm{a}}$ & $0.97 \pm 0.08$ & $0.036^{b}$ & $9.3 \pm 4.1$ & $0.015^{c}$ & $12.0 \pm 5.3$ & $0.022^{\mathrm{d}}$ \\
\hline Absent $(n=20)$ & 13 & 7 & & 9 & 11 & & $0.90 \pm 0.13$ & & $6.1 \pm 3.0$ & & $7.9 \pm 4.2$ & \\
\hline \multicolumn{13}{|l|}{ Acinar pattern } \\
\hline Present $(n=11)$ & 6 & 5 & 0.88 & 0 & 11 & $0.012^{\mathrm{e}}$ & $1.00 \pm 0.00$ & $0.001^{\dagger}$ & $9.3 \pm 4.7$ & 0.104 & $11.9 \pm 5.9$ & 0.165 \\
\hline Absent $(n=27)$ & 14 & 13 & & 11 & 16 & & $0.91 \pm 0.12$ & & $6.9 \pm 3.3$ & & $8.7 \pm 4.2$ & \\
\hline \multicolumn{13}{|l|}{ Nuclear contours } \\
\hline Smooth $(n=18)$ & 11 & 7 & 0.321 & 7 & 11 & 0.2 & $0.92 \pm 0.12$ & 0.373 & $6.4 \pm 3.7$ & 0.142 & $8.5 \pm 5.2$ & 0.191 \\
\hline Irregular $(n=20)$ & 9 & 11 & & 4 & 16 & & $0.95 \pm 0.10$ & & $8.5 \pm 3.9$ & & $11.0 \pm 5.0$ & \\
\hline \multicolumn{13}{|l|}{ Nucleoli } \\
\hline Conspicuous $(n=13)$ & 5 & 8 & 0.207 & 1 & 12 & $0.037^{9}$ & $0.99 \pm 0.01$ & $0.002^{\mathrm{h}}$ & $9.4 \pm 4.0$ & $0.031^{i}$ & $12.5 \pm 5.3$ & $0.020^{\mathrm{j}}$ \\
\hline Inconspicuous $(n=25)$ & 15 & 10 & & 10 & 15 & & $0.91 \pm 0.13$ & & $6.4 \pm 3.4$ & & $8.3 \pm 4.3$ & \\
\hline \multicolumn{13}{|l|}{ Intranuclear inclusions } \\
\hline Present $(n=14)$ & 6 & 8 & 0.357 & 4 & 10 & 0.969 & $0.94 \pm 0.11$ & 0.916 & $6.4 \pm 2.9$ & 0.121 & $8.2 \pm 3.8$ & 0.093 \\
\hline Absent $(n=24)$ & 14 & 10 & & 7 & 17 & & $0.93 \pm 0.12$ & & $8.5 \pm 4.4$ & & $11.3 \pm 5.7$ & \\
\hline \multicolumn{13}{|l|}{ Nuclear grooves } \\
\hline Present $(n=23)$ & 10 & 13 & 0.162 & 6 & 17 & 0.63 & $0.95 \pm 0.10$ & 0.423 & $7.4 \pm 3.4$ & 0.603 & $9.5 \pm 4.3$ & 0.442 \\
\hline Absent $(n=15)$ & 10 & 5 & & 5 & 10 & & $0.92 \pm 0.13$ & & $8.1 \pm 4.8$ & & $11.0 \pm 6.7$ & \\
\hline
\end{tabular}

The abbreviations are the same as in Tables 1 and 2 ; ${ }^{a-j}$ Statistical significance was defined as $p<0.05$; $P$ values of $<0.05$ were considered to indicate statistical significance Table 3: The relationships between the cytological and radiological features in ADC patients.

and perfusion parameters would provide complementary functional information, because the 18F-FDG uptake was correlated with Ki67, while BF was correlated with MVD. The results of our previous study [12] which revealed a significantly positive correlation between the Ki67 labeling index and the SUV max were consistent with their finding. Our study suggests that the cytological factors solid type ADC with predominant 3D clusters and conspicuous nucleoli with a necrotic background predicted the presence of a solid nodule on CT and a high SUV max on PET, and that SQCC has the same radiological features.
The present study is associated with some limitations. First, the patient population was relatively small in comparison to similar studies. In particular, Only one tumor was cytomorphologically classified as papillary according to the IASLC/ATS/ERS classification. Secondly, patient selection was biased based on the condition that a cytological specimen must be obtained by a preoperative bronchoscopic biopsy. As a result, there were no pure GGO lesions (is considered to be difficult to obtain tissue fragments from such lesions by bronchoscopy). 
Citation: Tanaka R, Sakamoto N, Suzuki H, Tachibana K, Takei H, et al. (2017) The Cytomorphological Characteristics of Non-small Cell Lung Cancer are Associated with Its Radiological Features. J Cytol Histol 8: 482. doi: 10.4172/2157-7099.1000482

Page 5 of 5

\section{Conclusion}

The preoperative cytomorphological findings might predict the value of radiological features on CT and PET examinations. These features might also provide information about the degree of pathological invasiveness or biological malignancy of the tumor. In the near future, these findings might be useful for decision-making in relation to treatment strategies such as lobectomy or limited resection and may have applications in personalized medicine.

\section{Conflicts of Interest}

The authors declare no conflicts of interest in association with the present study. This research did not receive any specific grant from funding agencies in the public, commercial, or not-for-profit sectors.

\section{References}

1. Travis WD, Brambilla E, Nicholson AG, Yatabe Y, Austin JH, et al. (2015) The 2015 World Health Organization Classification of Lung Tumors: Impact of Genetic, Clinical and Radiologic Advances Since the 2004 Classification. J Thorac Oncol 10: 1243-1260.

2. Travis WD, Brambilla E, Noguchi M, Nicholson AG, Geisinger KR, et al. (2011) International association for the study of lung cancer/american thoracic society/ european respiratory society international multidisciplinary classification of lung adenocarcinoma. J Thorac Oncol 6: 244-285.

3. Yoshiya T, Mimae T, Tsutani Y, Tsubokawa N, Sasada S, et al. (2016) Prognostic Role of Subtype Classification in Small-Sized Pathologic NO Invasive Lung Adenocarcinoma. Ann Thorac Surg 102: 1668-1673.

4. Hung JJ, Yeh YC, Jeng WJ, Wu KJ, Huang BS, et al. (2014) Predictive value of the international association for the study of lung cancer/American Thoracic Society/European Respiratory Society classification of lung adenocarcinoma in tumor recurrence and patient survival. J Clin Oncol 32: 2357-2364.

5. Murakami S, Ito H, Tsubokawa N, Mimae T, Sasada S, et al. (2015) Prognostic value of the new IASLC/ATS/ERS classification of clinical stage IA lung adenocarcinoma. Lung Cancer 90: 199-204.

6. Rodriguez EF, Monaco SE, Dacic S (2013) Cytologic subtyping of lung adenocarcinoma by using the proposed International Association for the Study of Lung Cancer/American Thoracic Society/European Respiratory Society (IASLC/ATS/ERS) adenocarcinoma classification. Cancer Cytopathol 121: 629-637.

7. Rodriguez EF, Dacic S, Pantanowitz L, Khalbuss WE, Monaco SE (2015) Cytopathology of pulmonary adenocarcinoma with a single histological pattern using the proposed International Association for the Study of Lung Cancer/
American Thoracic Society/European Respiratory Society (IASLC/ATS/ERS) classification. Cancer Cytopathol 123: 306-317.

8. Higashi K, Ueda Y, Arisaka Y, Sakuma T, Nambu Y, et al. (2002) 18F-FDG uptake as a biologic prognostic factor for recurrence in patients with surgically resected non-small cell lung cancer. J Nucl Med 43: 39-45.

9. Nakamura H, Hirata T, Kitamura H, Nishikawa $\mathrm{J}$ (2009) Correlation of the standardized uptake value in FDG-PET with the expression level of cell-cyclerelated molecular biomarkers in resected non-small cell lung cancers. Ann Thorac Cardiovasc Surg 15: 304-310.

10. Tanaka R, Horikoshi H, Nakazato Y, Seki E, Minato K, et al. (2009) Magnetic Resonance Imaging in Peripheral Lung Adenocarcinoma: Correlation With Histopathologic Features. J Thorac Imaging 24: 4-9.

11. Tanaka R, Horikoshi H, Yoshida T, Nakazato Y, Seki E, et al. (2011) Diffusionweighted magnetic resonance imaging in differentiating the invasiveness of small lung adenocarcinoma. Acta Radiol 52: 750-755.

12. Tanaka R, Nakazato Y, Horikoshi H, Tsuchida S, Yoshida T, et al. (2013) Diffusion-weighted imaging and positron emission tomography in various cytological subtypes of primary lung adenocarcinoma. Clin Imaging 37: 876-883.

13. Mountain CF (1997) Revisions in the International System for Staging Lung Cancer. Chest 111: 1710-1717.

14. Sigel CS, Moreira AL, Travis WD, Zakowski MF, Thornton RH, et al. (2011) Subtyping of non-small cell lung carcinoma: a comparison of small biopsy and cytology specimens. J Thorac Oncol 6: 1849-1856.

15. Billah S, Stewart J, Staerkel G, Chen S, Gong Y, et al. (2011) EGFR and KRAS mutations in lung carcinoma: molecular testing by using cytology specimens. Cancer Cytopathol 119: 111-117.

16. Nizzoli R, Tiseo M, Gelsomino F, Bartolotti M, Majori M, et al. (2011) Accuracy of fine needle aspiration cytology in the pathological typing of non-small cell lung cancer. J Thorac Oncol 6: 489-493.

17. Chiu CH, Yeh YC, Lin KH, Wu YC, Lee YC, et al. (2011) Histological Subtypes of Lung Adenocarcinoma Have Differential F-Fluorodeoxyglucose Uptakes on the Positron Emission Tomography/Computed Tomography Scan. J Thorac Oncol 6: 1697-1703.

18. Shimosato Y, Noguchi M (2004) Pulmonary neoplasms. In: Mills SE, ed. Sternberg' Diagnostic Surgical Pathology, (4th edn), Philadelphia, PA Lippincott Williams \& Wilkins: 1173-1222.

19. Sauter AW, Winterstein S, Spira D, Hetzel J, Schulze M, et al. (2017) Multifunctional profiling of non-small cell lung cancer using 18F-FDG PET/CT and volume perfusion CT. J Nucl Med 53: 521-529. 